\title{
Numerical modelling of the impact of flood wave cyclicality on the stability of levees
}

\author{
Anna Franczyk ${ }^{1, a}$, Maciej Dwornik ${ }^{1}$ and Andrzej Leśniak ${ }^{1}$ \\ ${ }^{1}$ AGH - University of Science and Technology, Faculty of Geology, Geophysics and Environmental Protection, al Mickiewicza 30, 30-059 \\ Krakow, Poland
}

\begin{abstract}
Sensitivity analysis applied to the flooding process is discussed in the paper. The analysis was done as part of the ISMOP project devoted to elaborating and designing a complex system for embankment monitoring and threat forecasting. The analysis was performed using selected geotechnical parameters that describe embankment state. It was shown that the sensitivity analysis method is very practical for detecting places where the largest vertical displacement and pore pressure distribution are observed. The sensitivity analysis was carried out for a single flood wave numerical experiment as well as for a double successive flood wave experiment. Comparison of the results allowed us to detect the places where the biggest differences in total relative sensitivity values are observed. Plots of these differences can help to indicate the particular places within the embankment that are the most influenced by successive flood waves and should be especially examined during field experiments as part of the ISMOP project.
\end{abstract}

\section{Introduction}

Reliable flood defence is an important issue for the maintenance of the security of any country. This is a key issue for not only lowland areas and environmental protection, but is also essential for densely populated areas with a high degree of industrialisation. A commonly used method of flood protection is river embankments. Without embankments, most countries would be regularly inundated during seasonal high water levels or other sudden weather phenomena resulting in intensive precipitation. Therefore, much attention is paid nowadays to issues related to the design, construction, and maintenance of river embankments. In addition to visual inspection and modern sensor technology, a lot of work is also carried out to update levee state assessments during high water conditions [1-4]. The ISMOP project [5] is an example of such an experiment conducted currently in Poland. The main aim of the ISMOP project is to research complex systems for embankment monitoring and threat forecasting [6-7]. For the purpose of this project, an experimental embankment was constructed in order to measure the impact of given flood wave scenarios on the river embankment. Considering the fact that experiments conducted during the tests cannot cover all possible scenarios for the development of flood waves, several numerical models were constructed to discover the parameters that have the most impact on flood waves. A sensitivity analysis method previously used for uncertainty analysis in a real geotechnical problem [8] was used to indicate the most significant parameters of flood waves in terms of embankment state.

\section{Sensitivity analysis}

Sensitivity analysis methods can be used in a wide range of scientific areas including decision-making, communication, and estimation or analysis of relationships between input and output variables in order to increase understanding of complicated systems. The other field of application is model development, including simplifying or calibrating models and testing them for validity and accuracy. Sensitivity analysis can be applied in many different ways [9-10]; however, various analyses may not produce the same results [11].

In this work, a sensitivity analysis method was applied to the problem of determining the flood wave parameters that have most influence on embankment state. The analysis can be divided into three separate stages in which values of sensitivity ratio, sensitivity score, and relative sensitivity are calculated respectively.

The sensitivity ratio $\eta_{\mathrm{SR}}$ can be described as the percentage change in model output divided by the unit change of the input variable according to Peschl [12]:

$$
\eta_{S R} \frac{\left[\frac{f\left(x_{L, G}\right)-f\left(x_{m}\right)}{f\left(x_{m}\right)}\right]}{\left[\frac{x_{L, G}-x_{m}}{x_{m}}\right]}
$$

where: $f\left(x_{m}\right)$ is the reference value of model output obtained after using the reference value $x_{m}$ of the given parameter and $f\left(x_{L, G}\right)$ is the value of the output variable

\footnotetext{
${ }^{\mathrm{a}}$ Corresponding author: franczyk@geol.agh.edu.pl
} 
obtained after using the changing value of the given input parameter $x_{L, G}$

For each considered parameter, four sensitivity ratios are computed and can be separated into two categories: local and global. For the local category, input parameter value $x_{L}$ is varied within a small interval of a random set. In the global sensitivity ratio, the input value of a given parameter $x_{\mathrm{G}}$ is varied across the whole range of a random set (fig. 1).

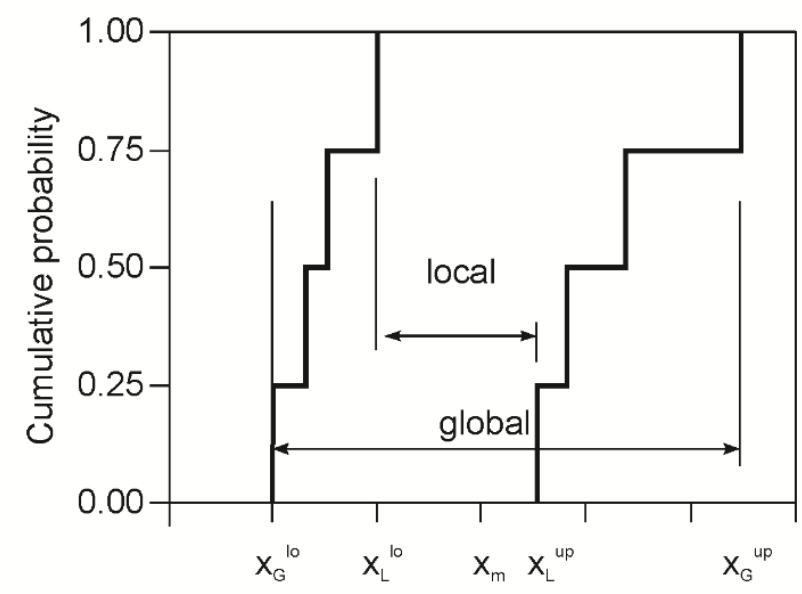

Figure 1. Local and range intervals and schematic representation of sensitivity calculated from sensitivity ratio. Modified after [12].

Sensitivity score $\eta_{S S}$ is a more robust method of evaluating the uncertainty of a given model. It is obtained by normalising and weighting the sensitivity ratio value in an input parameter. It can be written with the following expression:

$$
\eta_{S S}=\eta_{S R} \frac{\left(\max x_{G}-\min x_{G}\right)}{x}
$$

The normalisation procedure makes the sensitivity score independent of the input value units of a given parameter. Sensitivity score $\eta_{S S, i} i=1,2 . . N$ is calculated for all $\mathrm{N}$ number of basic parameters being considered.

The total relative sensitivity $\alpha\left(x_{i}\right)$ for each input variable is computed as a summation of all sensitivity scores (local and range) for each input parameter $\eta_{S S, I}$ on the respective results such as displacement, forces, pore pressure, or factor of safety. It can be written as (3):

$$
\alpha\left(x_{i}\right)=\frac{\sum \eta_{S S, i}}{\sum_{i=1}^{N} \sum \eta_{S S, i}}
$$

The total relative sensitivity can be used to determine which parameters have great impact on the safety factor if a threshold value is determined. Usually a threshold value between $5 \%$ and $10 \%$ is considered appropriate [13].

The sensitivity analysis of the geotechnical construction was conducted using a single value of specific parameters such as the safety factor of the slope [13-14] or displacement at a few points within the considered construction [15]. Sensitivity analysis is also used to indicate changes in parameter values during development of the modelled process [8]. Recently, sensitivity analysis conducted on all computational nodes of a numerical model has been introduced [16]. In this paper, sensitivity analysis calculations conducted for the parameters that describe the evolution of the flood wave for all computational points of the river embankment are presented.

\section{Flood wave cyclicality modelling}

Numerical modelling of the impact of flooding on embankment state was performed using FLAC, a twodimensional explicit finite difference program. The software allows the performing of the coupled mechanical-fluid and flow-thermal processes used in embankment stability modelling [17].

\subsection{Description of the geological model}

Numerical calculations were performed using a numerical model of the experimental embankment built for the ISMOP project. The geometry of the assumed numerical model that is consistent with the cross- section through the experimental embankment is depicted in (fig. 2).

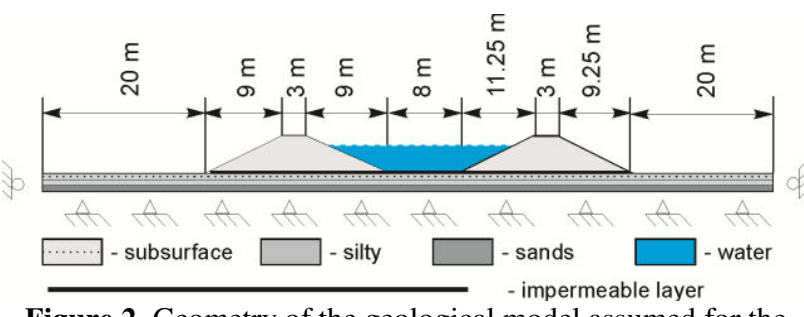

Figure 2. Geometry of the geological model assumed for the numerical calculation.

Material parameters for the assumed model are presented in the table below (Tab. 1).

\begin{tabular}{|c|c|c|c|c|c|}
\hline & $\begin{array}{c}\text { Symm } \\
\text { etric } \\
\text { levee }\end{array}$ & $\begin{array}{c}\text { Asymm } \\
\text { etric } \\
\text { levee }\end{array}$ & $\begin{array}{c}\text { Subsu } \\
\text { rface }\end{array}$ & Silty & Sand \\
\hline $\begin{array}{c}\text { Density } \\
{[\mathrm{g} / \mathrm{cm} 3]}\end{array}$ & 2.08 & 2.07 & 2.10 & 1.89 & 1.83 \\
\hline $\begin{array}{c}\text { Cohesion } \\
{[\mathrm{kPa}]}\end{array}$ & 16.9 & 24.1 & 10.3 & 13.7 & 9.75 \\
\hline $\begin{array}{c}\text { Friction } \\
{[\mathrm{deg}]}\end{array}$ & 37.6 & 35.6 & 32.9 & 22.5 & 35.8 \\
\hline $\begin{array}{c}\text { Bulk module } \\
{[\mathrm{MPa}]}\end{array}$ & 10.1 & 7.25 & 7.25 & 16.2 & 36.3 \\
\hline $\begin{array}{c}\text { Shear module } \\
{[\mathrm{MPa}]}\end{array}$ & 6.04 & 3.35 & 3.43 & 6.63 & 21.8 \\
\hline Porosity & $27 \%$ & $27 \%$ & $27 \%$ & $40 \%$ & $35 \%$ \\
\hline $\begin{array}{c}\text { Permeability } \\
{[\mathrm{m} / \mathrm{s}]}\end{array}$ & 1.18 & $\begin{array}{c}1.80 \\
\mathrm{x} 10^{-4}\end{array}$ & $\begin{array}{c}1.49 \\
\mathrm{x} 10^{-4}\end{array}$ & $\begin{array}{c}1.32 \\
\mathrm{x} 10^{-4}\end{array}$ & $\begin{array}{c}5.50 \\
\mathrm{x} 10^{-4}\end{array}$ \\
\hline $\begin{array}{c}\text { Specific heat } \\
{[\mathrm{J} / \mathrm{Kg} \mathrm{K}]}\end{array}$ & \multicolumn{5}{|c|}{840 for all geological medium and } \\
\hline $\begin{array}{c}\text { Thermal } 189 \text { for fluid } \\
\text { conductivity } \\
{[\mathrm{W} / \mathrm{mK}]}\end{array}$ & \multicolumn{5}{|c|}{1.6 for all geological medium and } \\
\hline
\end{tabular}

Table 1. Material parameters 


\subsection{Description of the sensitivity analysis of the flooding process.}

Development of the flood wave experiment conducted during the ISMOP project can be described by five independent parameters: rate of water level increase (fig. 3a), time of high water persistence (fig. 3b), rate of water level decrease (fig. 3c), interval between successive flood waves (fig. 3d), and height of flood waves (fig. 3e).

a)

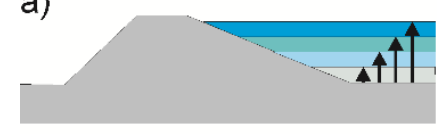

b)

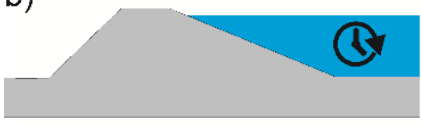

c)

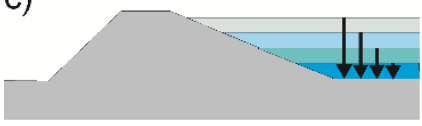

d)

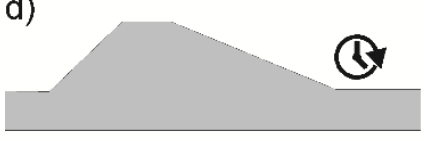

e)

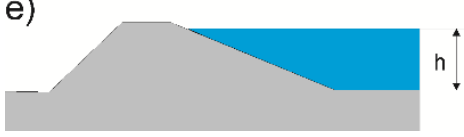

Figure 3. Parameters of flood wave: ascent (a), high plateau (b), descent (c), low plateau (d) and height of water (e).

During the sensitivity analysis, two types of flood process were examined: a single flood wave, and flood waves comprising two successive flood waves. Two intervals of parameters were determined independently. The first set was determined by analysis of historical floods in Poland in the basin of the Vistula River where the experimental embankment was built [18]. The second was assumed according to the ISMOP project documentation that applied certain limited values to the rate of water level changes and the height of water level (experimental knowledge). Parameters of flood wave water regarded as basic variables are summarised in table 2. The reference value of each flood wave parameter was assumed as a mean of the values of two assumed intervals.

\begin{tabular}{|c|c|c|c|c|}
\hline & \multicolumn{2}{|c|}{$\begin{array}{c}\text { Experimental } \\
\text { investigation }\end{array}$} & \multicolumn{2}{c|}{ Expert knowledge } \\
\hline & $\min$ & $\max$ & $\min$ & $\max$ \\
\hline $\begin{array}{c}\text { ascent } \\
{[\mathrm{h}]}\end{array}$ & 24 & 72 & 36 & 60 \\
\hline $\begin{array}{c}\text { high plateau } \\
{[\mathrm{h}]}\end{array}$ & 12 & 120 & 24 & 84 \\
\hline $\begin{array}{c}\text { descent } \\
{[\mathrm{h}]}\end{array}$ & 54 & 126 & 66 & 90 \\
\hline $\begin{array}{c}\text { low plateau } \\
{[\mathrm{h}]}\end{array}$ & 24 & 144 & 48 & 96 \\
\hline $\begin{array}{c}\text { water height } \\
{[\mathrm{m}]}\end{array}$ & 3 & 4 & 3.25 & 3.75 \\
\hline
\end{tabular}

Table 2. Basic variables for material parameters (input values).

\subsection{Numerical calculations.}

For the sensitivity analysis of the flooding process described above, more than twenty deterministic models are needed. In each model the lower or upper limit of the local and range interval of each parameter was adopted. The value of the remaining four parameters was fixed with reference values. Results of numerical modelling were pore pressure distribution and vertical displacement obtained for each computational node of the assumed numerical model. Values from all 21 flooding process development scenarios were then processed in order find which of the five basic parameters are most significant and contribute most to output variability.

\section{Results of the sensitivity analysis of the flood wave parameters.}

Sensitivity analysis of the flood wave parameters was carried out for a single flood wave and double successive flood waves on the river embankment. Figures $4,5,7$, and 8 show the results of the analysis obtained for all computational nodes, indicating the impact of the basic flood parameters on pore pressure distribution and vertical displacement within the embankment. These plots correspond to the value of the sensitivity score (equation no 2) described above. The results of sensitivity analysis conducted for pore pressure distribution and vertical displacements that correspond to the total relative sensitivity (equation no 3) are also presented (figures 6 and 9). In the presented plots, the sensitivity score and total relative sensitivity values less than $10 \%$ were discarded.

\subsection{Sensitivity results after single flood wave.}

The numerical modelling of the flooding process was carried out with different duration for each of the 21 scenarios. Duration of the flood wave modelling varied from 9 to 14 days. The results of sensitivity analysis using the sensitivity score for pore pressure and vertical displacement distribution and total relative sensitivity are presented in figures 4,5 , and 6 respectively.

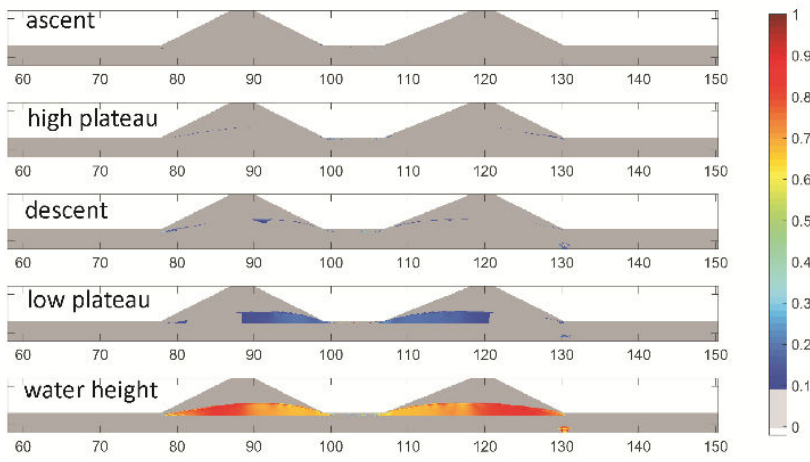

Figure 4. Sensitivity score values of flood wave parameters for the pore pressure distribution. 


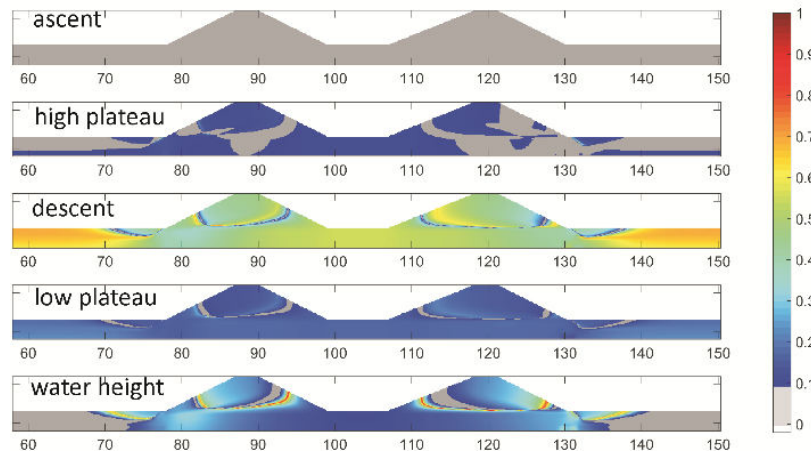

Figure 5. Sensitivity score values of flood wave parameters for the vertical displacement distribution.

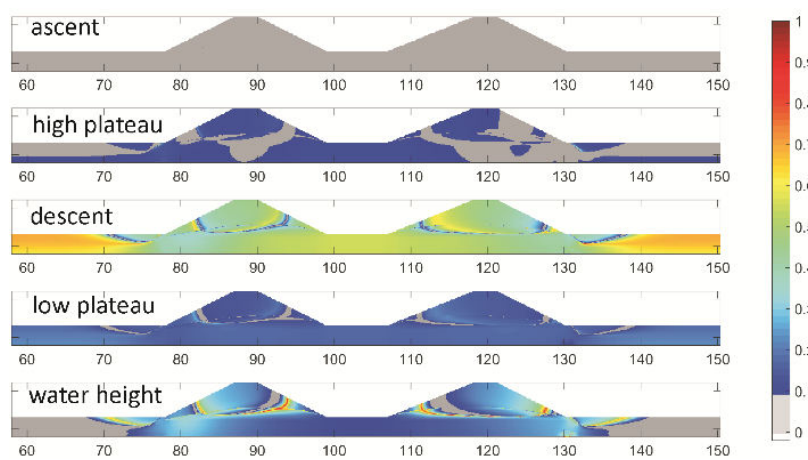

Figure 6. The total relative sensitivity values of flood wave parameters.

For the distribution of sensitivity score values obtained both for pore pressure field and vertical displacement modelling, no effects of the rate of water level increase can be observed. This is also reflected in the total relative sensitivity values, which are actually less than 5\%. The sensitivity score obtained for the results of the pore pressure modelling show that the most significant flood wave parameters are water height and time interval after successive flood waves (low plateau). However, there are some embankment areas where also the rate of the water level decreasing and the duration of the high plateau are significant values. The same calculation performed for vertical displacement of the embankment shows regions where the impact of the rate of water level decrease and the duration of the high plateau can be observed. Figure 6 shows that when a $10 \%$ threshold value is accepted, there are four most significant parameters that should be used when constructing scenarios for embankment stability analysis: duration time of high water level, rate of high water level fall, time interval between successive flood waves, and water level.

\subsection{Sensitivity results after double successive flood wave.}

We extended our research of the sensitivity analysis of the flood wave parameters to the case of a double flood wave. Such analysis allows us to estimate the real impact of the low water plateau duration parameter on the distribution of pore pressure and vertical displacement values within the embankment. As in the case of a single flooding process, different flood wave modelling duration times were applied for all 21 scenarios. In this case, the minimum scenario time of the flood process was 18 days, compared to the maximum of 28 days. The results of sensitivity analysis using the sensitivity score for pore pressure and vertical distribution and total relative sensitivity for a double flood wave cycle are presented in figures 7,8 and 9 respectively.

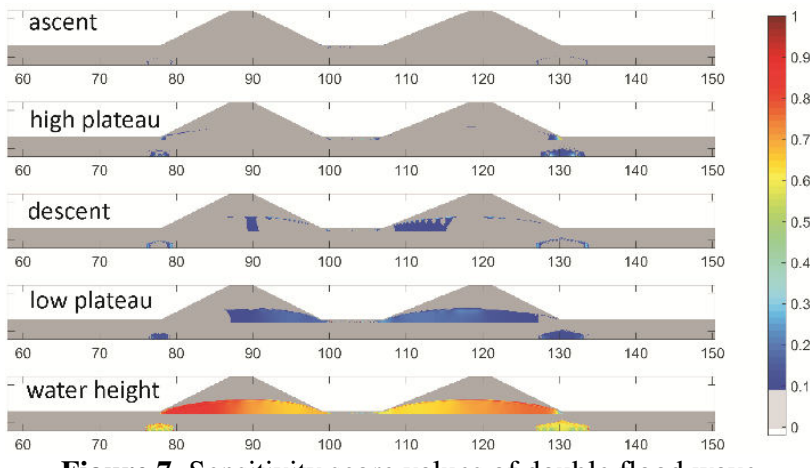

Figure 7. Sensitivity score values of double flood wave parameters for pore pressure distribution.

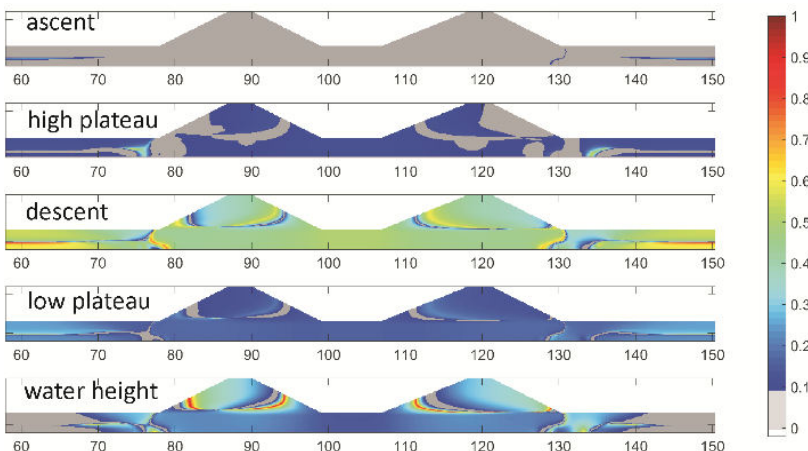

Figure 8. Sensitivity score values of double flood wave parameters for vertical displacement distribution.

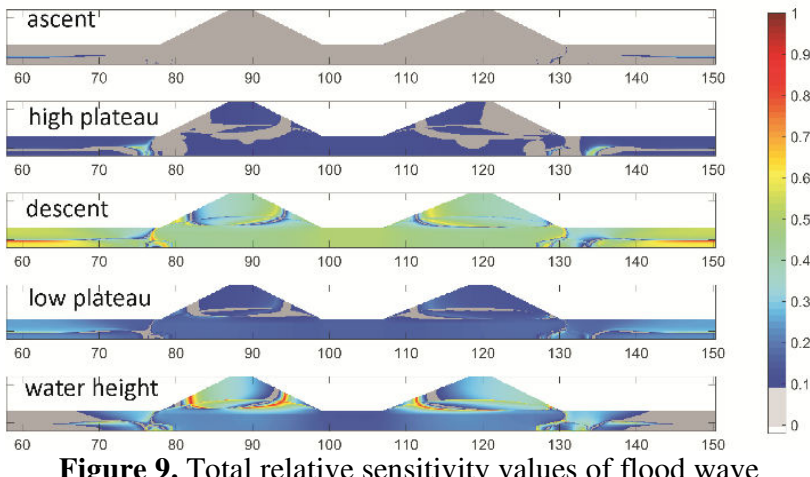

Figure 9. Total relative sensitivity values of flood wave parameters obtained for double flood wave cycles.

The results obtained for the application of sensitivity analysis to double successive flood wave modelling also indicate that the rate of water level increase is insignificant for embankment state. Its value can be fixed and should not be included in the determination of flood wave scenarios. The sensitivity analysis of the impact of a double flood wave indicated the greater influence of water level decrease rate and high plateau duration on pore pressure results than was calculated for a single flood wave. This is a consequence of existing saturation caused by the first flood wave. In figure 10 , differences between the total sensitivity values 
computed for single and double flood waves are presented. The differences were plotted for all four significant flooding process parameters.

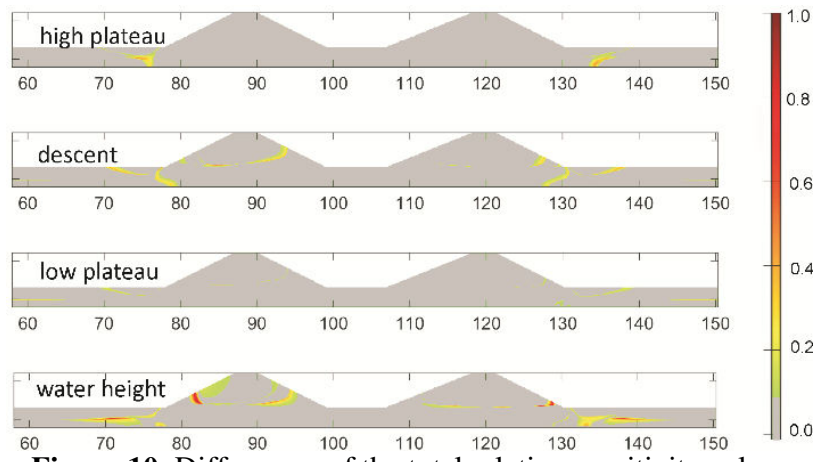

Figure 10. Differences of the total relative sensitivity values between single and double flood wave cycles.

The plots of differences show that all four parameters are sensitive to residual saturation. The places where differences are observed are generally in the same part of the embankment. Of all four significant flooding process parameters, the plot of water level reaches the highest values. The differences in total relative water level sensitivity computed for single and double flood waves are also visible on the largest areas of the embankment relative to other parameters.

\section{Conclusions}

In this paper, sensitivity analysis was applied to the problem of the influence of flooding processes on embankment state.

It was shown that the sensitivity analysis method is very practical for dealing with detection of places in the embankment where the biggest impact on vertical displacement and pore pressure distribution are observed.

The sensitivity analysis conducted for single flood wave scenarios and for double successive flood waves allowed the same conclusions to be drawn about the significance of the flood wave parameters. Both types of scenarios (single flood, double flood) indicated four significant parameters: the duration of high water level, the rate of high water level fall, time interval between successive flood waves, and water level. As is presented in this paper, these parameters should be used in the construction of scenarios for embankment stability analysis.

The sensitivity analysis also allowed the biggest differences in total relative sensitivity values to be detected. The differences plot can help to indicate areas within the embankment that are most exposed to successive flood waves and should be especially examined as part of the ISMOP project.

\section{Acknowledgements.}

This work is financed by the National Centre for Research and Development (NCBiR), Poland, project PBS1/B9/18/2013 - (no 180535).

This work was partly supported by AGH - University of Science and Technology, Faculty of Geology, Geophysics and Environmental Protection, as a part of statutory research project.

\section{References}

1. Van, m. A., Zwanenburg, C., Koelewijn, A. R. and van Lottum, H. (2009). Evaluation of Full Scale Levee Stability Tests at Booneschans and Corresponding Centrifuge Tests. Proc. 17th Int. Conf. on Soil Mech. and Geot. Eng., Alexandria, Egypt, pp. 2048-2051.

2. Erdbrink, C.D., Krzhizhanovskaya, V.V. and Sloot, P.M.A., (2012), Controlling flow-induced vibrations of flood barrier gates with data-driven and finite-element modelling. Comprehensive Flood Risk Management - Klijn \& Schweckendiek (eds) (C) Taylor \& Francis Group, London, pp. 425434.

3. Krzhizhanovskaya V. V., Shirshov G. S., Melnikova N. B., Belleman R. G., Rusadi F. I., Broekhuijsen B. J,. Gouldby B. P., Lhomme J., Baliś B., Bubak M., Pyayt A. L., Mokhov I. I., Ozhigin A. V., Lang B. and Meijer R. J. (2011). Flood early warning system: design, implementation and computational modules, Procedia Computer Science 4, 106-115.

4. Radzicki, K., Bonelli, S. (2012). Monitoring of the suffusion process development using thermal analysis performed with IRFTA model, w Proc. of 6th ICSE, 593-600.

5. Moscicki J.W., Bania, G., Cwiklik, M. and Borecka A. (2014). DC resistivity studies of shallow geology in the vicinity of Vistula river flood bank in Czernichów Village (near Kraków in Poland). Studia Geotechnica et Mechanica. 36 (1), 63-70

6. Pięta, A., Lupa, M., M., C., Piórkowski, A., and Leśniak, A. (2014). A model of a system for stream data storage and analysis dedicated to sensor networks of embankment monitoring. Computer Information Systems and Industrial Management, Lecture Notes in Computer Science. Berlin: Springer Berlin Heidelberg, 8838. pp. 514-525.

7. Chuchro, M., Lupa, M., Pięta, A., Piórkowski, A., and Leśniak, A. (2014). A concept of time windows length selection in stream databases in the context of sensor networks monitoring. Advances in databases and information systems and associated satellite events. ADBIS 2014 Advances in Intelligent Systems and Computing. Springer International Publishing. pp. 173-174.

8. Pięta A., Krawiec K. (2015). Random set method application to flood embankment stability modeling. Procedia Computer Science, 51, 2668-2677.

9. Clemson, B., Tang, Y., Pyne, J. and Unal, R. (1995). Efficient methods for sensitivity analysis, System Dynamics Review 11, 31-49.

10. Lomas, K.J. and Eppel, H. (1992). Sensitivity analysis techniques for building thermal simulation programs, Energy and Buildings 19, 21-44.

11. Iman R. L. and Helton, J. C. (1988). An Investigation of Uncertainty and Sensitivity Analysis Techniques for Computer Models. Risk Analysis 8, 71-90.

12. Peschl G. M. (2004). Reliability analysis in geotechnics with the random set finite element method. PhD thesis. Graz: Graz University of Technology, (unpublished). 
13. Shen, H., and Abbas, S. (2013). Rock slope reliability analysis based on distinct element method and random set theory. Int. J. of Rock Mech. and Mining Sci, 15-22.

14. Pilecki, Z., Stanisz, J., Krawiec, K., Woźniak, H., and Pilecka, E. (2014). Numerical stability analysis of slope with use of Random Set Theory. Zeszyty Naukowe IGSMiE PAN 86, 5 - 17.

15. Schweiger, H. F., and Peschl, G. M. (2005). Reliability analysis in geotechnics with the random set finite element method. Computers and Geotechnics, $422-435$.

16. Dwornik, M., Krawiec, K., Pięta, A., Leśniak, A. (2015) Numerical and experimental stability analysis of earthen levees. IAMG 2015 : the 17th annual conference of the International Association for Mathematical Geosciences : Freiberg, Germany, Short Abstract: pp.163-164, Full Paper (DVD): pp. 857-864

17. Itasca Consulting Group, I. (2011). FLAC Fast Lagrangian Analysis of Continua and FLAC/Slope User's Manual.

18. Kret E. (2015). ISMOP Internal Raport, Task 2.2. 